ARTIGO ORIGINAL ORIGINAL ARTICLE

\section{Custo-efetividade de trimetazidina no tratamento de angina estável em pacientes diabéticos não respondedores a betabloqueadores, nitratos e bloqueadores do canal de cálcio}

\author{
Cost-effectiveness of trimetazidine for stable angina \\ treatment in non-responder diabetics after beta \\ blockers, nitrates and calcium channel blockers
}

Luiz Antonio Machado Cesar ${ }^{1,2}$, Camila Pepe ${ }^{3}$, Roberta Arinelli3

DOI: 10.21115/JBES.v9.n2.p164-76

\section{RESUMO}

Palavras-chave:

angina estável, angina pectoris, avaliação de custo-efetividade, diabetes mellitus, doença das coronárias, trimetazidina

\section{Keywords:}

angina, stable, angina pectoris, cost-effectiveness evaluation, diabetes mellitus, coronary disease, trimetazidine
Objetivo: Avaliar o custo-efetividade da trimetazidina (TMZ) associada ao tratamento convencional (diurético, ácido acetilsalicílico [AAS], betabloqueador, inibidores da enzima de conversão da angiotensina (IECA), nitrato, estatina, digitálico e antagonista dos canais de cálcio) versus tratamento convencional isolado no tratamento de pacientes diabéticos com angina estável não respondedores a betabloqueadores, nitratos e bloqueadores do canal de cálcio sob a perspectiva do Sistema Público de Saúde (SUS). Métodos: Foi elaborado um modelo de Markov, com ciclos de 1 ano, que acompanhou os pacientes ao longo do curso natural da doença até o final de suas vidas. Dados clínicos foram obtidos a partir de revisão da literatura. Custos unitários foram extraídos de bases de dados oficiais. Apenas custos médicos diretos foram contemplados. Custos e benefícios foram descontados a uma taxa de 5\% ao ano. Desfecho clínico foi expresso como episódio de hospitalização por angina pectoris (EHAP) evitado. Análise de sensibilidade univariada foi realizada para determinar os parâmetros de maior influência nos resultados, variando-os em mais ou menos 20\% comparado ao cenário base. Resultados: $O$ tratamento com TMZ mostrou maior benefício comparado ao tratamento convencional e gerou uma Razão de Custo-Efetividade Incremental de R\$ 7.344,96 por EHAP evitado. O parâmetro de maior impacto no resultado foi o número de episódios de angina pectoris. Apesar do alto impacto, este não alterou o resultado da análise, mantendo-o custo-efetivo. Conclusão: TMZ associada ao tratamento convencional mostrou ser uma alternativa eficaz, segura e custo-efetiva para o tratamento de pacientes diabéticos com angina estável não respondedores a betabloqueadores, nitratos e bloqueadores do canal de cálcio. 
review. Unit costs were extracted from official databases. Only direct medical costs were included. Costs and benefits were discounted at a rate of 5\% per year. Outcome were expressed as hospitalization due to angina pectoris (HAP) avoided. Univariate sensitivity analysis was performed to determine the parameters of greatest influence on the results, varying them by plus or minus 20\% compared to the baseline scenario. Results: Treatment with TMZ showed greater benefit compared to conventional treatment and generated an Incremental Cost-Effectiveness Ratio of USD 2,225.75 per HAP avoided (1USD = 3.30BRL). The parameter with the greatest impact on outcome was the number of angina pectoris episodes. Despite the high impact, it did not change the result, keeping it cost-effective. Conclusion: TMZ associated with conventional treatment has been shown to be an effective, safe and cost-effective alternative for the treatment of diabetic patients with stable angina non-responders to beta blockers, nitrates and calcium channel blockers.

\section{Introdução}

A doença arterial coronariana (DAC) é considerada uma das principais causas de óbito no mundo ocidental e, no Brasil, também representa uma das maiores causas de hospitalização e custos aos sistemas de saúde (Alves et al., 2010; Bocchi et al., 2012). A angina pectoris é sua principal manifestação clínica, sendo decorrente da isquemia do miocárdio, que ocorre quando oxigênio insuficiente é fornecido para o músculo cardíaco. Caracteriza-se por dor ou desconforto no tórax, epigástrio, mandíbula, ombro, dorso ou membros superiores, desencadeados principalmente pelo esforço físico ou estresse e aliviados com repouso ou medicamentos (Alves et al., 2010; Cesar et al., 2014). A angina pode ser classificada como estável ou instável, de acordo com o aparecimento dos sintomas. Tipicamente, a forma estável surge em situações de esforço e desaparece com o repouso. Já a instável se apresenta principalmente como angina em repouso, angina de aparecimento recente ou angina progressiva (Cesar et al., 2014).

A angina afeta cerca de 112 milhões de pessoas no mundo (Werdan et al., 2015). Na Austrália, está presente em mais de 353 mil habitantes e causa 72 mil admissões hospitalares anualmente (Wee et al., 2015). No Reino Unido, a prevalência da angina estável é de aproximadamente 2,1 milhões de pessoas (Coleman et al., 2015). Nos Estados Unidos, a cada ano, há 3,4 milhões de pessoas com 40 anos ou mais de idade com a doença (Benjamin et al., 2017). Estima-se que o Brasil tenha, pelo menos, 900 mil habitantes com angina pectoris e cerca de 18 mil novos casos da doença ao ano (Sociedade Brasileira de Cardiologia, 2004).

Nos pacientes com DAC, alguns subgrupos podem apresentar gravidade ainda maior, entre eles os portadores de diabetes mellitus (DM). O DM é um importante fator de risco para eventos cardiovasculares futuros em pacientes com DAC. A taxa de mortalidade cardiovascular em diabéticos com DAC é duas vezes maior que a de pacientes não diabéticos (Rosano et al., 2005). Em geral, a presença de DM faz com que os pacientes manifestem a ocorrência de doenças cardiovasculares 15 anos antes do esperado. Homens e muIheres diabéticos tipos 1 e 2, com idades respectivamente superiores a 40 e 50 anos geralmente apresentam risco de eventos coronarianos $>2 \%$ ao ano (Sociedade Brasileira de Diabetes, 2015). De acordo com dados do Sistema de Informação Sobre Mortalidade levantados por Barreto et al., 2007 (Barreto et al., 2007), foi estimado um total de 237.946 óbitos $(8,8 \%)$ relacionados ao DM em adultos acima de 30 anos, residentes nas regiões Sul e Sudeste do Brasil, entre os anos de 1999 e 2003. Em 4,2\% dos adultos, o diabetes foi causa básica e em 4,6\% causa associada. Doenças cardiovasculares contabilizaram 54,5\% das causas básicas de óbito quando DM foi uma causa associada (Barreto et al., 2007).

Em um estudo econômico (Straka et al., 2009) que avaliou os custos associados ao manejo de eventos cardiovasculares em pacientes diabéticos versus não diabéticos, a população diabética apresentou maior permanência hospitalar e custos médicos diretos mais elevados durante a hospitalização e o acompanhamento. O custo incremental para o grupo de diabéticos foi de US\$ 10.131 por paciente em período de três anos, quando comparado ao grupo de não diabéticos.

Os objetivos fundamentais do tratamento da angina estável incluem: 1) prevenir o infarto agudo do miocárdio (IAM) e reduzir a mortalidade cardiovascular; 2) reduzir os sintomas e a ocorrência da isquemia miocárdica, propiciando melhor qualidade de vida (QV). Entre as opções terapêuticas encontram-se orientação dietética e de atividade física, e tratamentos medicamentoso, cirúrgico e intervencionista (César et al., 2004; Fox et al., 2006).

A trimetazidina (TMZ) é um derivado da piperazina, usada como agente antianginoso, que promove a inibição seletiva da enzima envolvida na betaoxidação de tiolase 3-acetil-coenzima A de cadeia longa. Seus benefícios têm sido atribuídos à preservação dos níveis intracelulares de trifosfato de adenosina (ATP) e da fosfocreatina, com o mesmo oxigênio residual (Kantor et al., 2000), redução da acidose (Mody et al., 1998), sobrecarga de cálcio (Kay et al., 1995) e acúmulo de radicais livres induzidos pela isquemia (Maridonneau-Parini \& Harpey, 1985) e preservação das membranas celulares.

Atualmente, não existe protocolo clínico do Ministério da Saúde para o tratamento da angina estável. No entanto, as diretrizes da Sociedade Brasileira de Cardiologia para o tratamento da doença coronária estável recomendam a utilização de TMZ para pacientes com angina estável sintomática em 
uso de betabloqueadores isoladamente ou associados a outros agentes antianginosos, para angina estável e disfunção de VE associado à terapia clínica otimizada, e para angina estável durante procedimentos de revascularização miocárdica, percutânea ou cirúrgica.

A partir da identificação da relevância dos pacientes diabéticos com DAC, o presente estudo teve como objetivo avaliar o custo-efetividade da TMZ adjuvante ao tratamento convencional, composto por diuréticos, ácido acetilsalicílico (AAS), betabloqueadores, inibidores da enzima conversora da angiotensina (IECA), nitratos, estatinas, digitálicos e antagonistas dos canais de cálcio versus tratamento convencional da angina estável em pacientes diabéticos não respondedores a betabloqueadores, nitratos e bloqueadores do canal de cálcio.

\section{Métodos}

\section{Tipo de estudo e intervenções}

A análise de custo-efetividade foi desenvolvida com base em uma estrutura de cadeia de Markov. Para o desenvolvimento do modelo matemático de decisão, a seguinte comparação foi considerada: tratar os pacientes diabéticos com angina estável não respondedores a betabloqueadores, nitratos e bloqueadores do canal de cálcio, com TMZ de forma adjuvante ao tratamento convencional (diurético, AAS, betabloqueador, IECA, nitrato, estatina, digitálico e antagonista dos canais de cálcio) versus tratamento convencional apenas.

\section{Estrutura do modelo}

O modelo de Markov elaborado acompanhou os pacientes diabéticos com angina estável ao longo do curso natural da doença até o final de suas vidas, e a transição dos pacientes pelos seguintes estados de saúde foi considerada: angina estável sem evento, hospitalização por doenças isquêmicas do coração, hospitalização por angina pectoris, angina estável pós-hospitalização, angina estável pós-angina pectoris, morte por causas isquêmicas e morte por outras causas. Foram utilizados ciclos de um ano, ou seja, a cada ano eram possíveis transições dos pacientes entre os estados de saúde. A Figura 1 representa a estrutura do modelo.

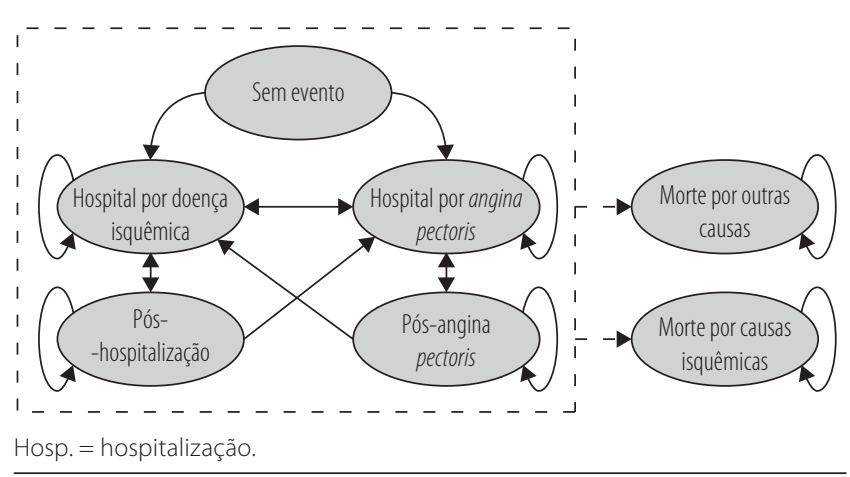

Figura 1. Estrutura do modelo de Markov.
No modelo, o paciente diabético com angina estável inicia o tratamento com TMZ adjuvante ao tratamento convencional ou mantém apenas o tratamento convencional após ser considerado não respondedor a betabloqueadores, nitratos e bloqueadores de canal de cálcio no estado de "angina estável sem evento". Ao longo dos ciclos do Markov, os pacientes podem permanecer neste estado, necessitar de uma hospitalização por doença isquêmica do coração, hospitalização por angina pectoris, morrer por causas isquêmicas ou por outras causas. Os pacientes que sofrem uma hospitalização por doença isquêmica do coração ou hospitalização por angina pectoris podem sobreviver ao evento e passar para o estado de "angina estável pós-hospitalização" ou "angina estável pós-angina pectoris", ou ainda podem morrer por causas isquêmicas ou por outras causas. Enquanto vivos, os pacientes podem sofrer novas hospitalizações, morrer por causas isquêmicas ou por outras causas.

Os resultados comparativos das estratégias de tratamento foram avaliados pela razão de custo-efetividade incremental (RCEI), definida para duas alternativas de tratamentos específicos como o custo adicional proporcionado pela alternativa em análise dividido pelo ganho adicional em saúde alcançado por esta. Foi considerado como limiar de custo-efetividade o valor do Produto Interno Bruto (PIB) per capita no Brasil em 2016 de R\$ 30.407 (Instituto Brasileiro de Geografia e Estatística (IBGE), 2016). O desfecho principal considerado na análise foram casos de hospitalização evitados. Portanto, a RCEl foi calculada visando identificar o custo necessário para se evitar uma hospitalização.

Análise de sensibilidade univariada foi realizada, com o objetivo de determinar os parâmetros de maior influência em relação aos resultados do modelo econômico apresentado. Para isso, todos os parâmetros foram variados em mais ou menos 20\% em relação ao cenário base.

\section{Revisão da literatura}

Os desfechos clínicos e dados epidemiológicos utilizados no modelo econômico foram coletados por meio de revisão sistemática da literatura científica. A revisão e análise crítica da literatura foram realizadas com o objetivo de avaliar o número de episódios e a redução de angina nos pacientes tratados (efetividade e segurança) com TMZ combinada à terapia convencional, quando comparada ao padrão de tratamento adotado pelo Sistema Único de Saúde (SUS), ou seja, sem TMZ. As bases de dados utilizadas foram MEDLINE via PubMed, Cochrane Library, LILACS e Centre for Reviews and Dissemination (CRD). Foram incluídos metanálises, revisões sistemáticas, ensaios clínicos, estudos observacionais e estudos de mundo real que tivessem avaliado a TMZ no tratamento da angina estável em pacientes diabéticos não respondedores a betabloqueadores, nitratos e bloqueadores dos canais de cálcio, publicados até maio de 2016. Na busca não foram utilizados limites temporais ou de idiomas. Dois revisores realizaram a 
busca nas bases de dados e selecionaram os estudos para inclusão na revisão. Planejou-se, inicialmente, que, nos casos em que não houvesse consenso, um terceiro revisor seria consultado sobre a elegibilidade e ficaria responsável pela decisão final. De acordo com as Diretrizes Metodológicas para Elaboração de Pareceres Técnico-Científicos do Ministério da Saúde (Ministério da Saúde (Brasil), Secretaria de Ciência-Tecnologia e Insumos Estratégicos. Departamento de Ciência e Tecnologia, 2014), os estudos incluídos na revisão foram analisados conforme cada questionário recomendado para cada tipo de estudo, permitindo a avaliação sistemática da "força" da evidência, principalmente na identificação de potenciais vieses e seus impactos na conclusão do estudo.

\section{Uso de recursos e custos}

Os recursos de saúde considerados se referem aos custos inerentes à perspectiva pública e resumem-se a medicamentos, hospitalizações por doenças isquêmicas do coração e acompanhamento dos pacientes após uma hospitalização.

O tratamento com TMZ foi realizado com uma dose de $35 \mathrm{mg}$ duas vezes ao dia ao longo de todo o horizonte de vida do paciente e foi custeado de acordo com o preço proposto pelo fabricante para incorporação, com um desconto de 33\% em relação ao preço máximo de venda ao governo obtido da lista de preços da Câmara de Regulação do Mercado de Medicamentos (CMED) (Ministério da Saúde - Brasil. Agência Nacional de Vigilância Sanitária - ANVISA, 2016).

Além do tratamento medicamentoso com TMZ, foram adicionados os custos da terapia convencional (diuréticos, AAS, betabloqueadores, IECA, nitratos, estatinas, digitálicos e antagonistas dos canais de cálcio). Os custos unitários dos medicamentos foram obtidos do Banco de Preços em Saúde e a dose média utilizada, de acordo com a recomendação de bula dos medicamentos (Ministério da Saúde - Brasil, 2015).

Caso os pacientes sofressem uma hospitalização por doença isquêmica do coração ou angina pectoris, havia custos associados, de acordo com o valor médio por internação do DATASUS, para o período de maio de 2015 a maio de 2016 (Ministério da Saúde - Brasil. Departamento de Informática do SUS - DATASUS, 2016).

Também foi contabilizado o custo de acompanhamento com consultas e exames para pacientes que tenham sofrido ou não hospitalizações prévias de acordo com o Sistema de Gerenciamento da Tabela de Procedimentos, Medicamentos e OPM do SUS (SIGTAP) (Ministério da Saúde - Brasil. DATASUS - Departamento de Informática do SUS, 2016).

\section{Resultados}

\section{Clínicos}

Por meio de uma revisão sistemática da literatura, a eficácia e a segurança do uso de TMZ combinada à terapia conven- cional foram avaliadas no tratamento da angina estável em pacientes diabéticos não respondedores a betabloqueadores, nitratos e bloqueadores dos canais de cálcio, quando comparadas ao padrão de tratamento adotado pelo SUS. Após a realização da busca nas bases de dados, 53 títulos (incluindo duplicatas) foram localizados. Aplicados os critérios de elegibilidade, dois revisores selecionaram nove estudos para leitura na íntegra. Desses, foram incluídos nove artigos completos, sendo oito ensaios clínicos (Fragasso et al., 2003; Marazzi et al., 2007; Ribeiro et al., 2007; Rosano et al., 2003; Szwed et al., 1999; Thrainsdottir et al., 2004; Xu et al., 2014; Zhao et al., 2013) e um estudo observacional pós-comercialização (Rodríguez Padial et al., 2005), a fim de embasar as evidências científicas do uso de TMZ. O fluxograma de seleção de artigos está apresentado no Apêndice 1.

Para o modelo econômico, optou-se por utilizar os dados do estudo de Xu et al., 2014, por se tratar de um estudo com os resultados apresentados para o grupo de pacientes em tratamento convencional, podendo ou não ter recebido TMZ adjuvante (Xu et al., 2014). As características dos pacientes avaliados no estudo foram utilizadas para definir a idade inicial no modelo e a porcentagem de uso de cada medicamento do tratamento padrão (Tabela 1).

O estudo de Xu et al., 2014, apresenta o número de hospitalizações por doenças isquêmicas, eventos de angina pectoris e mortalidade por causas isquêmicas em dois anos (Xu et al., 2014). As probabilidades em dois anos foram convertidas para anuais. A fórmula de conversão utilizada está apresentada a seguir, e os resultados obtidos estão detalhados na Tabela 2. As probabilidades de mortalidade por outras causas foram aplicadas a pacientes com idade inicial média de 69 anos.

Tabela 1. Características dos pacientes na linha de base, nos grupos TMZ (trimetazidina + tratamento convencional) e controle (tratamento convencional)

\begin{tabular}{lccc}
\hline Variável & TMZ & Controle & Valor de $\mathbf{p}$ \\
\hline Pacientes ( $\mathrm{n})$ & 255 & 255 & - \\
\hline Idade (anos) & 68,94 & 68,52 & 0,153 \\
\hline Mulheres $(\mathrm{n})$ & 83 & 81 & 0,850 \\
\hline Medicamentos concomitantes $(\mathrm{n})$ & & & \\
\hline AAS & 255 & 255 & - \\
\hline Clopidogrel & 255 & 255 & - \\
\hline Estatinas & 255 & 255 & - \\
\hline Betabloqueadores & 173 & 187 & 0,174 \\
\hline IECA ou BRA & 124 & 108 & 0,155 \\
\hline Nitratos & 196 & 208 & 0,190 \\
\hline
\end{tabular}

$\mathrm{AAS}=$ ácido acetilsalicílico; $\mathrm{TMZ}=$ trimetazidina; $\mathrm{IECA}=$ inibidor da enzima conversora da angiotensina; BRA = bloqueador de receptor de angiotensina. Fonte: Xu et al., 2014. 
Tabela 2. Ocorrência de hospitalização e morte por causas isquêmicas nos grupos TMZ (trimetazidina + tratamento convencional) e controle (tratamento convencional)

\begin{tabular}{lccc}
\hline Eventos & TMZ & Controle & Valor de $\mathbf{p}$ \\
\hline IAM e AVE & 25 & 27 & - \\
\hline Eventos (n) & 9,80 & 10,59 & - \\
\hline Em 2 anos (\%) & 5,03 & 5,44 & - \\
\hline Anual (\%) & & & \\
\hline Revascularização & 75 & 90 & 0,156 \\
\hline Eventos (n) & 29,41 & 35,29 & - \\
\hline Em 2 anos (\%) & 15,98 & 19,56 & - \\
\hline Anual (\%) & & & \\
\hline Angina pectoris & 72 & 96 & 0,024 \\
\hline Eventos (n) & 28,24 & 37,65 & - \\
\hline Em 2 anos (\%) & 15,29 & 21,04 & - \\
\hline Anual (\%) & & & \\
\hline Recorrência de angina pectoris & 102 & 130 & 0,01 \\
\hline Eventos (n) & 40,00 & 50,98 & - \\
\hline Em 2 anos (\%) & 22,54 & 29,99 & - \\
\hline Anual (\%) & & & \\
\hline Mortes por causas isquêmicas & 24 & 29 & 0,468 \\
\hline Eventos (n) & 9,41 & 11,37 & - \\
\hline Em 2 anos (\%) & 4,8 & 5,86 & - \\
\hline Anual (\%) & & & \\
\hline
\end{tabular}

$\mathrm{TMZ}=$ trimetazidina; $\mid \mathrm{AM}$ = infarto agudo do miocárdio; $\mathrm{AVE}=$ acidente vascular encefálico.

Fonte: Xu et al., 2014

Equação 1: Fórmula de conversão das probabilidades para taxas anuais

Probabilidade_anual $=1-(1-$ Probabilidade_24meses $)\left({ }^{(2 / 24}\right)$

As probabilidades de mortalidade segmentadas por ano foram retiradas do Instituto Brasileiro de Geografia e Estatística (IBGE) para idades até 80 anos (Instituto Brasileiro de Geografia e Estatística - IBGE, 2010). A partir dessa idade, as probabilidades anuais foram projetadas considerando-se uma projeção linear do logaritmo neperiano da probabilidade de mortalidade a partir de 45 anos. Essa probabilidade é elevada com o aumento da idade dos pacientes ao longo do modelo, como detalhado no Apêndice 2.

As probabilidades anuais foram ajustadas excluindo da taxa geral de mortalidade as mortes por doenças isquêmicas do coração, equivalentes a 8,8\% de todos os óbitos de acordo com dados do Sistema de Informações em Mortalidade do DATASUS (Instituto Brasileiro de Geografia e Estatística IBGE, 2013). Foi utilizada a seguinte fórmula para o ajuste das probabilidades de mortalidade anuais excluindo-se as mortes por doenças isquêmicas do coração:

Equação 2: Fórmula para ajuste das probabilidades de mortalidade anuais

Probabilidade $=$ Probabilidade_12meses ${ }^{*}$ mort_não_isq
Onde:

Probabilidade: Probabilidade de mortalidade ajustada; Probabilidade_12meses: Probabilidade de mortalidade esperada em 12 meses; mort_não_isq: mortalidade geral excluindo as mortes por doenças isquêmicas do coração, equivalente a 91,2\% neste caso, sendo igual a 100\% menos 8,8\% dos óbitos associados a doenças isquêmicas do coração (Instituto Brasileiro de Geografia e Estatística - IBGE, 2013).

A Figura 2 apresenta os resultados das extrapolações realizadas, sendo estes os resultados utilizados na composição do modelo.

\section{Econômicos}

Os custos associados à utilização de TMZ e tratamento convencional pelo ciclo de um ano são apresentados na Tabela 3 e na Tabela 4, respectivamente. $\bigcirc$ Apêndice 3 mostra maior detalhamento do tratamento convencional.

Os custos de acompanhamento para pacientes que sofreram uma hospitalização por doença isquêmica e para aqueles sem evento prévio estão apresentados na Tabela 5 e, de forma detalhada, no Apêndice 3.

Os custos de tratamento medicamentoso, tratamento de eventos cardiovasculares e acompanhamento de pacientes com ou sem eventos prévios foram ponderados pelo percentual de pacientes em cada estado de saúde a cada ciclo da análise. A Figura 3 apresenta a composição total dos custos do modelo.

Os resultados de custo da comparação de TMZ adjuvante ao tratamento convencional com o tratamento convencional estão apresentados na Tabela 6, e os resultados comparativos de efetividade estão apresentados na Tabela 7. Como pode ser observado na Figura 4, é esperada uma redução das hospitalizações por angina estável com a incorporação da TMZ.

O uso da TMZ proporcionou ainda um aumento da sobrevida média esperada dos pacientes tratados. Os resultados de custo-efetividade, expressos como custo incremental por hospitalização por angina pectoris evitada, estão apresentados na Tabela 8.

Pode-se observar que o custo total de tratamento com TMZ em combinação com o tratamento convencional é superior ao custo do tratamento convencional no horizonte de tempo de cinco anos. Esse resultado é direcionado principalmente pelo custo de o medicamento ser parcialmente compensado pelo menor custo com hospitalizações por doenças isquêmicas. Em relação à eficácia, a TMZ apresentou um resultado melhor que o grupo com tratamento convencional, reduzindo o número esperado de casos de hospitalizações por angina pectoris e doenças isquêmicas e aumentando o número médio de anos vividos. Chega-se, portanto, a um resultado de maior custo e maior efetividade. 
Sem eventos

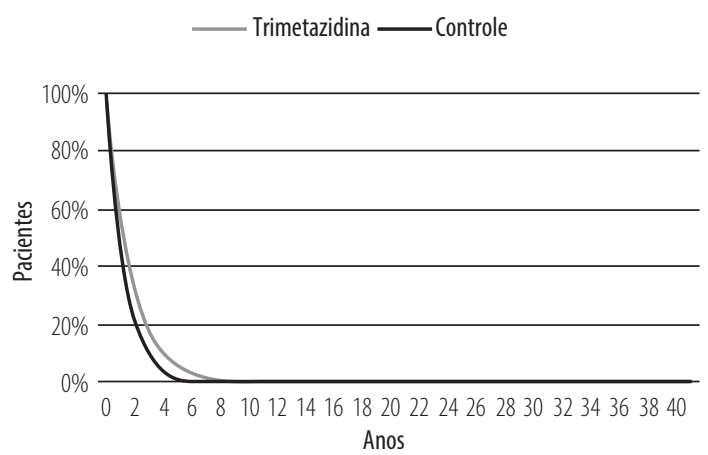

Morte por causas isquêmicas

— Trimetazidina __ Controle

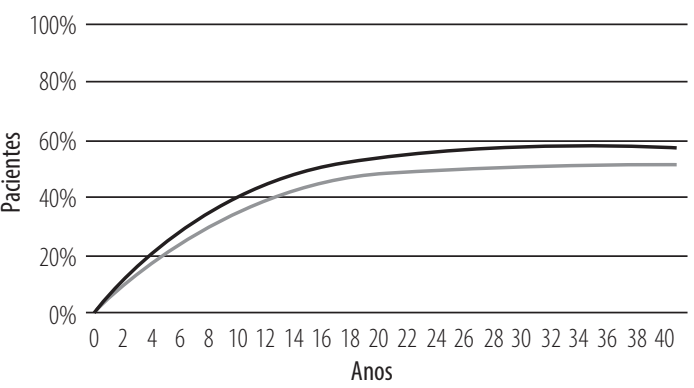

Evento cardiovascular

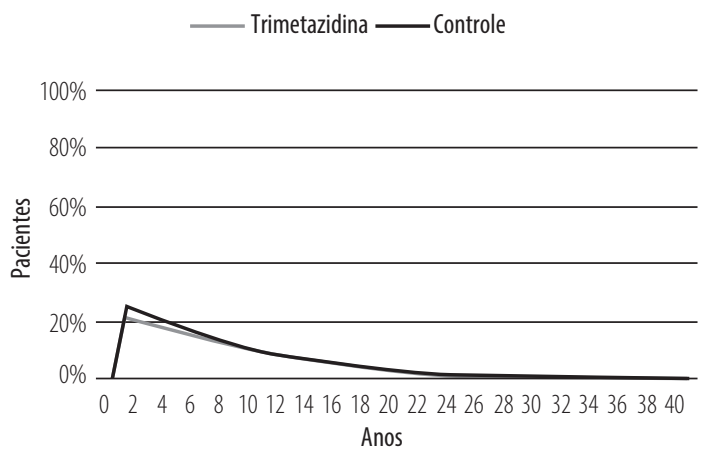

Outras mortes
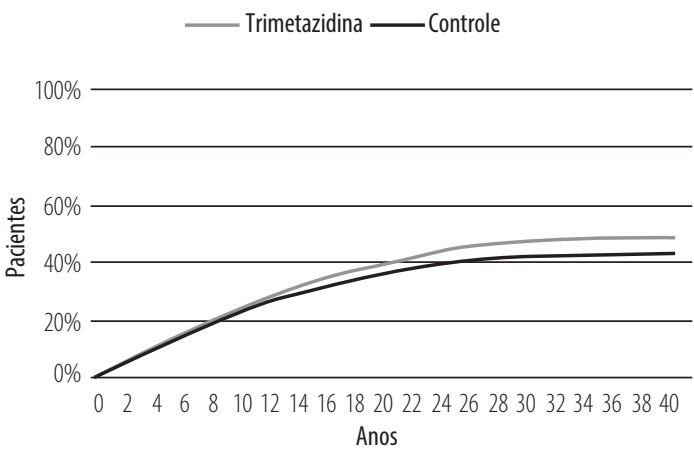

Figura 2. Percentual de pacientes sem evento, com evento cardiovascular, com morte por causa isquêmica ou morte por outra causa, nos grupos TMZ (trimetazidina + tratamento convencional) e controle (tratamento convencional), em horizonte temporal de 40 anos (extrapolação de dados de efetividade).

Tabela 3. Custo anual de tratamento com trimetazidina

\begin{tabular}{lcccccc}
\hline Medicamento & Nome Comercial & $\begin{array}{c}\text { PMVG } \\
\text { (R\$, ICMS 18\%) }\end{array}$ & $\begin{array}{c}\text { Preço } \\
\text { considerado* (R\$) }\end{array}$ & $\begin{array}{c}\text { Custo/ } \\
\text { dose (R\$) }\end{array}$ & $\begin{array}{c}\text { Doses/ano } \\
\text { Custo anual (R\$) }\end{array}$ \\
\hline TMZ 35 mg x 30 comp. & Vastarel MR & 40,43 & 21,00 & 0,70 & 720 & 504,00 \\
\hline TMZ 35 mg x 60 comp. & Vastarel MR & 80,86 & 42,00 & 0,70 & 720 & 504,00 \\
\hline
\end{tabular}

* Informado pela empresa fabricante. PMVG = preço máximo de venda ao governo (Câmera de Regulação do Mercado de Medicamentos - CMED); TMZ = trimetazidina.

Tabela 4. Custo anual do tratamento convencional

\begin{tabular}{lcc}
\hline Medicamentos & $\begin{array}{c}\text { Pacientes em uso (\%) } \\
\text { (Xu et al., 2014) }\end{array}$ & \begin{tabular}{c} 
Custo anual (R\$)* \\
\hline AAS
\end{tabular} \\
\hline Clopidogrel & 100 & 21,64 \\
\hline Estatinas & 100 & 129,04 \\
\hline Betabloqueadores & 70,59 & 991,25 \\
\hline Inibidores da ECA & 45,49 & 356,35 \\
\hline Antagonistas da & 45,49 & 26,41 \\
angiotensina II & & $4.972,23$ \\
\hline
\end{tabular}

AAS = ácido acetilsalicílico; ECA = enzima conversora da angiotensina.

* Baseado no Preço Máximo de Venda ao Governo (PMVG - ICMS18\%) fornecido pela Câmera de Regulação do Mercado de Medicamentos - CMED.

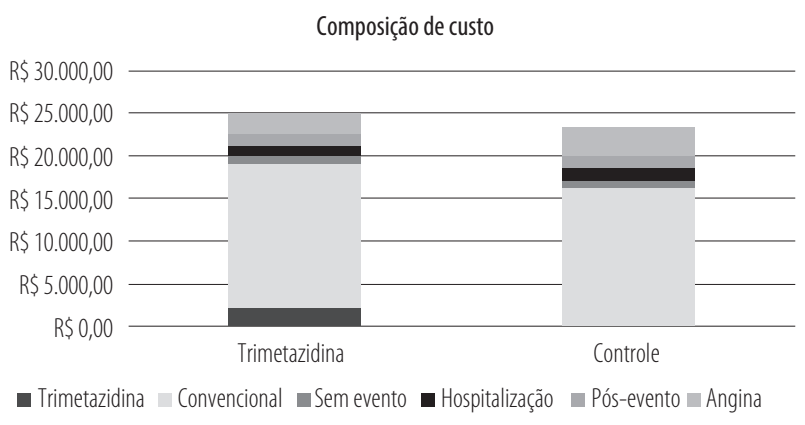

Figura 3. Composição de custos do modelo nos grupos TMZ (trimetazidina + tratamento convencional) e controle (tratamento convencional). 
Tabela 5. Resultados de custos para os grupos TMZ (trimetazidina + tratamento convencional) e controle (tratamento convencional)

\begin{tabular}{lccc}
\hline Tipos de custo & TMZ (R\$) & Controle (R\$) & Incremental (R\$) \\
\hline Medicamentos & $20.990,41$ & $18.013,08$ & $2.977,33$ \\
\hline TMZ & $2.514,50$ & 0,00 & $2.514,50$ \\
\hline Tratamento convencional & $18.475,91$ & $18.013,08$ & 462,83 \\
\hline Hospitalização por doença isquêmica & $1.381,29$ & $1.610,09$ & $-228,80$ \\
\hline Hospitalização por angina pectoris & $2.940,62$ & $4.006,63$ & $-1.066,01$ \\
\hline Seguimento pós-evento & $1.512,39$ & $1.518,09$ & $-5,70$ \\
\hline Seguimento sem evento & $1.129,20$ & 927,77 & 201,43 \\
\hline Total (sem desconto) & $27.953,91$ & $26.075,67$ & $1.878,24$ \\
\hline Total (com desconto) & $24.975,54$ & $23.290,81$ & $1.684,73$ \\
\hline
\end{tabular}

$\mathrm{TMZ}=$ trimetazidina.

Tabela 6. Resultados de efetividade

\begin{tabular}{|c|c|c|c|}
\hline Desfecho & TMZ & Controle & Incremental \\
\hline \multicolumn{4}{|c|}{ Episódios de hospitalização por angina pectoris } \\
\hline Sem desconto & 0,73 & 0,99 & $0,26^{*}$ \\
\hline Com desconto & 0,63 & 0,86 & $0,23^{*}$ \\
\hline \multicolumn{4}{|c|}{ Episódios de hospitalização por doença isquêmica } \\
\hline Sem desconto & 0,91 & 1,06 & $0,15^{*}$ \\
\hline Com desconto & 0,79 & 0,92 & $0,13^{*}$ \\
\hline \multicolumn{4}{|l|}{ Anos de vida } \\
\hline Sem desconto & 3,99 & 3,86 & 0,12 \\
\hline Com desconto & 3,48 & 3,38 & 0,11 \\
\hline
\end{tabular}

* Número de eventos evitados. TMZ = trimetazidina.

Tabela 7. Custo por hospitalização e acompanhamento

\begin{tabular}{lcc}
\hline Recursos de saúde & $\begin{array}{c}\text { Custo por hospitalização/ } \\
\text { acompanhamento (R\$) }\end{array}$ & Fonte \\
\hline Hospitalização por IC & $1.523,21$ & DATASUS - maio/15 a maio/16 \\
\hline Hospitalização por angina pectoris & $4.053,21$ & DATASUS - maio/15 a maio/16 \\
\hline Acompanhamento pós-hospitalização & 739,42 & Calculado \\
\hline Acompanhamento sem evento & 509,06 & Calculado \\
\hline
\end{tabular}

IC = insuficiência cardíaca.

Tabela 8. Resultados de custo-efetividade descontados no horizonte temporal de cinco anos

\begin{tabular}{lcccc}
\hline Desfecho & TMZ & Controle & Incremental \\
\hline Custo total (R\$) & $24.975,54$ & $23.290,81$ & $1.684,73$ \\
\hline Episódios de hospitalização por angina pectoris & 0,63 & 0,86 & 0,23 \\
\hline RCEI (R\$_/episódio de hospitalização por angina evitada) & & $7.344,96$ & \\
\hline
\end{tabular}

$\mathrm{RCEI}=$ razão de custo-efetividade incremental; TMZ = trimetazidina.

A RCEI calculada para os diferentes desfechos da análise resultou em $\mathrm{R} \$$ 7.344,96 por hospitalização por angina pectoris evitada (Tabela 8). Considerando-se que o custo adicional por hospitalização por angina pectoris evitada calculado está abaixo do limite de uma vez o PIB per capita no Brasil, igual a R\$ 28.876 em 2015, pode-se dizer que o tratamento com TMZ é custo-efetivo em comparação ao tratamento convencional somente (Instituto Brasileiro de Geografia e Estatística - IBGE, 2016). 


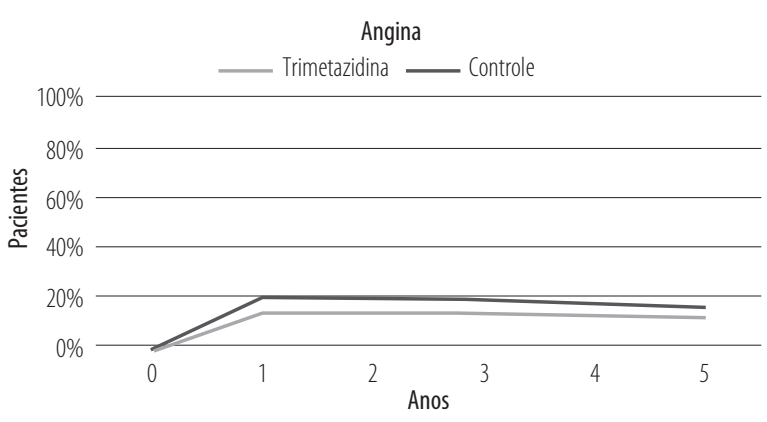

Figura 4. Percentual de pacientes hospitalizados por angina nos grupos TMZ (trimetazidina + tratamento convencional) e controle (tratamento convencional), em horizonte temporal de cinco anos.

\section{Análise de sensibilidade univariada}

Os resultados da análise de sensibilidade univariada estão apresentados na Figura 5. O parâmetro de maior impacto no resultado do modelo foi o número de episódios de angina pectoris em ambos os grupos. Apesar do alto impacto desse parâmetro, ele não alterou o resultado da análise, mantendo-o custo-efetivo. Os demais parâmetros não alteraram de maneira significativa os resultados da análise.

\section{Discussão}

O relevante impacto clínico e econômico das DAC nas populações de risco leva à necessidade de medidas comportamentais e medicamentosas que possam amenizar desfechos indesejáveis. Em estudo conduzido no Brasil, o custo anual médio estimado por paciente com cardiopatia isquêmica foi de $R \$ 2.733,00$ sob a perspectiva do SUS e de $R \$ 6.788,00$, para a perspectiva privada, com tendência a um maior custo naqueles com DM, quando comparados aos não diabéticos (Ribeiro et al., 2005). Em outro estudo nacional (Teich \& Araujo, 2011), a estimativa do custo direto associado à síndrome coronariana aguda em 2011 sob a perspectiva do SUS foi de R\$ 522.286.726 (0,77\% do orçamento total do sistema), e de $\mathrm{R} \$$ 515.138.617 para a saúde suplementar. Os custos indiretos totalizaram R\$2,8 bilhões, sob a perspectiva da sociedade. 0 custo total estimado para a síndrome coronariana aguda em 2011, incluindo custos diretos e indiretos, foi de $R \$ 3,8$ bilhões (Teich \& Araujo, 2011).

Uma análise da coorte de Framingham mostrou que indivíduos com DM não experimentaram os declínios necessários nos fatores de risco para doença cardiovascular a fim de superar seu risco basal aumentado e reforçou a necessidade de esforços para o controle agressivo de tais fatores entre os diabéticos (Preis et al., 2009). A taxa de mortalidade cardiovascular em pacientes diabéticos com DAC é duas a quatro vezes maior que a de pacientes não diabéticos (Benjamin et al., 2017; Rosano et al., 2005). Diabéticos respondem por cerca de um quarto de todos os procedimentos de revascularização coronária e apresentam resultados piores em comparação com pacientes não diabéticos (Berry et al., 2007; Donahoe et al., 2007). Pelo menos $68 \%$ dos pacientes diabéticos com mais de 65 anos de idade morrem por alguma forma de doença cardíaca (Benjamin et al., 2017).

A regulação do metabolismo da glicose é um marco importante no controle de fatores de riscos cardiovasculares. A captação de glicose nos músculos cardíacos e esqueléticos é inversamente proporcional aos níveis séricos de ácidos graxos livres (AGL). Além disso, novas evidências sugerem que níveis aumentados de AGL não só prejudicam a captação de glicose pelo coração e músculo esquelético, mas também

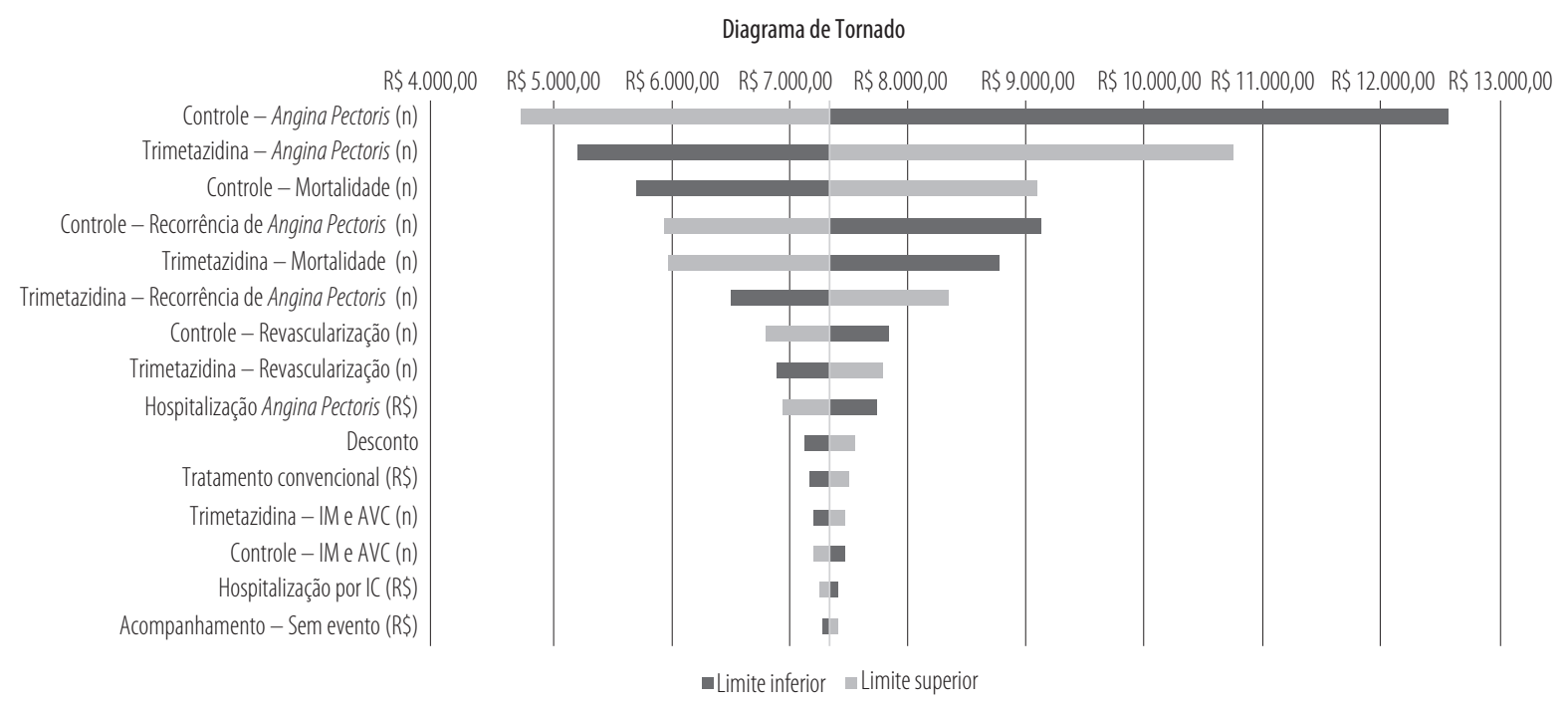

Figura 5. Diagrama de Tornado - Análise de sensibilidade univariada para o desfecho "Custo adicional por hospitalização por angina pectoris evitada". 
causam alterações no metabolismo do endotélio vascular, levando a aterogênese acelerada, alteração cardiovascular prematura, além de contribuir para o desenvolvimento de doença cardiovascular em pacientes diabéticos (Fragasso et al., 2009).

Os efeitos benéficos combinados da inibição parcial de AGL e metabolismo da glicose tornam o uso de fármacos com esse princípio particularmente atraente, especialmente nos pacientes cardiopatas que apresentam anormalidades do miocárdio e do metabolismo da glicose (Fragasso et al., 2009). Por meio de uma revisão sistemática da literatura, avaliaram-se a eficácia e segurança do uso da TMZ combinada à terapia convencional no tratamento da angina estável em pacientes diabéticos não respondedores a betabloqueadores, nitratos e bloqueadores dos canais de cálcio, quando comparada ao padrão de tratamento adotado pelo SUS. Nessa revisão foram incluídos nove artigos completos, sendo oito ensaios clínicos e um estudo observacional pós-comercialização, a fim de embasar as evidências científicas.

De acordo com os resultados demonstrados pelos estudos incluídos nessa análise, a TMZ em 20 mg administrada três vezes ao dia, que é equivalente à dosagem atualmente comercializada do medicamento de $35 \mathrm{mg}$ duas vezes ao dia, demonstrou ser bem tolerada e eficaz em pacientes diabéticos que não apresentaram controle com uso do tratamento padrão, controlando e melhorando diversos parâmetros cardíacos, bem como o metabolismo da glicose e a função endotelial (Fragasso et al., 2003; Marazzi et al., 2007; Ribeiro et al., 2007; Rodríguez Padial et al., 2005; Rosano et al., 2003; Szwed et al., 1999; Thrainsdottir et al., 2004; Xu et al., 2014; Zhao et al., 2013).

A análise de custo-efetividade realizada mostrou que o tratamento com TMZ tem maior benefício comparado ao tratamento convencional no cenário base da análise e gerou uma RCEl de R\$ 7.344,96 por episódio de hospitalização por angina pectoris evitada. Algumas limitações podem ser destacadas nesta análise. Para os dados clínicos de entrada utilizados, alguns ensaios clínicos apresentaram tamanho amostral reduzido, o que dificulta a extrapolação dos resultados para a população. Além disso, alguns não apresentavam braço comparador e apresentavam desenho de estudo aberto.

Um estudo de custo-efetividade foi conduzido na Grécia para avaliar TMZ como terapia complementar ao tratamento convencional em pacientes com angina crônica estável que não responderam adequadamente à terapia de primeira linha com betabloqueadores, nitratos ou antagonistas de canal de cálcio. A análise mostrou que o custo de TMZ adicionada ao tratamento convencional foi de $€ 1.755,57$ versus $€ 1.751,76$ para o tratamento convencional. A associação de TMZ gerou 0,6650 anos de vida ajustados pela qualidade (QALY) versus 0,6562 para o tratamento convencional, o que gerou uma RCEl de $€ 430,67$ por QALY ganho. O estudo concluiu que a adição de TMZ nesta população foi custo-efetiva.

Cabe ressaltar que essa avaliação econômica tem limitações inerentes à característica do estudo realizado. Por se tratar de um modelo baseado em projeção matemática, temos incertezas associadas. As incertezas e limitações são minimizadas com a aplicação de análises de sensibilidade que, neste estudo, comprovaram a robustez dos resultados do caso base. As limitações são inerentes às avaliações econômicas, como, por exemplo, a extrapolação dos dados de eficácia dos estudos clínicos para dados de efetividade.

\section{Conclusão}

Na análise desenvolvida sob a perspectiva do SUS, TMZ associada ao tratamento convencional mostrou ser uma alternativa eficaz, segura e custo-efetiva para o tratamento da angina estável na população de pacientes diabéticos com angina estável não respondedores a betabloqueadores, nitratos e bloqueadores do canal de cálcio.

\section{Referências bibliográficas}

Alves L, Cesar J, Horta B. Prevalence of Angina Pectoris in Pelotas (south of Brazil). Arq Bras Cardiol. 2010;95(2):179-85.

Barreto SM, Passos VM, Almeida SK, Assis TD. The increase of diabetes mortality burden among Brazilian adults. Rev Panam Salud Publica. 2007;22(4):239-45.

Benjamin EJ, Blaha MJ, Chiuve SE, Cushman M, Das SR, Deo R. Heart Disease and Stroke Statistics--2017 Update: A Report From the American Heart Association. Circulation. 2017;135(10):e146-e603.

Berry C, Tardif JC, Bourassa MG. Coronary Heart Disease in Patients With Diabetes. J Am Coll Cardiol. 2007;49(6):643-56.

Bocchi E, Marcondes-Braga F, Bacal F, Ferraz A, Albuquerque D, Rodrigues D. Atualização da Diretriz Brasileira de Insuficiência Cardíaca Crônica. Arq Bras Cardiol. 2012;98(1 supl. 1):1-33.

César LAM, Mansur AP, Armaganijan D, Amino JG, Sousa AC, Simão AF, et al. Diretrizes de doença coronariana crônica e angina estável. Arq Bras Cardiol. 2004;83(Suppl. 2):2-43.

Cesar L, Ferreira J, Armaganijan D, Gowdak L, Mansur A, Bodanese L, et al. Diretriz de Doença Coronária Estável. Arq Bras Cardiol. 2014;103(2 Supl. 2):1-59.

Coleman Cl, Freemantle N, Kohn CG. Ranolazine for the treatment of chronic stable angina: a cost-effectiveness analysis from the UK perspective. BMJ Open. 2015;5(11):e008861.

Donahoe SM, Stewart GC, McCabe CH, Mohanavelu S, Murphy SA, Cannon CP, et al. Acute Coronary Syndromes. JAMA. 2007;298(7):765-75.

Fox K, Garcia MA, Ardissino D, Buszman P, Camici PG, Crea F, et al. Guidelines on the management of stable angina pectoris: executive summary: The Task Force on the Management of Stable Angina Pectoris of the European Society of Cardiology. Eur Heart J. 2006;27(11):1341-81.

Fragasso G, Piatti Md PM, Monti L, Palloshi A, Setola E, Puccetti P. Short- and long-term beneficial effects of trimetazidine in patients with diabetes and ischemic cardiomyopathy. Am Heart J. 2003;146(5):E18.

Fragasso G, Salerno A, Spoladore R, Cera M, Montanaro C, Margonato A. Effects of metabolic approach in diabetic patients with coronary artery disease. Curr Pharm Des. 2009;15(8):857-62. 
Instituto Brasileiro de Geografia e Estatística (IBGE). (2013). Tábua de mortalidade 2013: Instituto Brasileiro de Geografia e Estatística. Tábuas completas de mortalidade 2013. Retrieved July 14, 2014. Disponível em: http://www.ibge.gov.br/home/estatistica/populacao/ tabuadevida/2012/.

Instituto Brasileiro de Geografia e Estatística (IBGE). (2016). Sala de Imprensa: contas nacionais trimestrais - Indicadores de volume e valores correntes. Disponível em: ftp://ftp.ibge.gov.br/Contas_Nacionais/ Contas_Nacionais_Trimestrais/Fasciculo_Indicadores_IBGE/pib-volval_201604caderno.pdf.

Instituto Brasileiro de Geografia e Estatística (IBGE). (2010). Tábua completa de mortalidade de 2010 - Ambos os sexos. Disponível em: http://www. ibge.gov.br/home/estatistica/populacao/tabuadevida/2013/defaulttab_ pdf.shtm.

Kantor PF, Lucien A, Kozak R, Lopaschuk GD. The antianginal drug trimetazidine shifts cardiac energy metabolism from fatty acid oxidation to glucose oxidation by inhibiting mitochondrial long-chain 3-ketoacyl coenzyme A thiolase. Circ Res. 2000;86(5):580-8.

Kay L, Finelli C, Aussedat J, Guarnieri C, Rossi A. Improvement of longterm preservation of the isolated arrested rat heart by trimetazidine: effects on the energy state and mitochondrial function. Am J Cardiol. 1995;76(6):45B-49B.

Marazzi G1, Wajngarten M, Vitale C, Patrizi R, Pelliccia F, Gebara O, et al. Effect of free fatty acid inhibition on silent and symptomatic myocardial ischemia in diabetic patients with coronary artery disease. Int J Cardiol. 2007;120(1):79-84.

Maridonneau-Parini I, Harpey C. Effect of trimetazidine on membrane damage induced by oxygen free radicals in human red cells. Br J Clin Pharmacol. 1985;20(2):148-51.

Ministério da Saúde (Brasil). (2015). Banco de Preços em Saúde. Disponível em: http://bps.saude.gov.br/bps/login.jsf.

Ministério da Saúde (Brasil). Agência Nacional de Vigilância Sanitária (ANVISA). (2016). Câmara de Regulação do Mercado de Medicamentos (CMED). Disponível em: http://portal.anvisa.gov.br/cmed.

Ministério da Saúde (Brasil). DATASUS - Departamento de Informática do SUS. (2016). Sistema de Gerenciamento da Tabela de Procedimentos, Medicamentos e OPM do SUS (SIGTAP). Disponível em: http://sigtap. datasus.gov.br.

Ministério da Saúde (Brasil). Departamento de Informática do SUS (DATASUS). (2016). Informações de Saúde - Assistência à Saúde - Procedimentos hospitalares por local de internação a partir de 2008. Disponível em: http://www2.datasus.gov.br/DATASUS/index.php?a.

Ministério da Saúde (Brasil). Secretária de Ciência, Tecnologia e Insumos Estratégicos. Departamento de Ciência e Tecnologia. (2014). Diretrizes metodológicas: elaboração de pareceres técnico-científicos. 4.ed. Brasília: Ministério da Saúde. Disponível em: http://doi.org/10.1007/ s13398-014-0173-7.

Mody FV, Singh BN, Mohiuddin IH, Coyle KB, Buxton DB, Hansen HW. Trimetazidine-induced enhancement of myocardial glucose utilization in normal and ischemic myocardial tissue: An evaluation by positron emission tomography. Am J Cardiol. 1998;82(5A):42K-49K.
Preis SR, Pencina MJ, Hwang SJ, D’Agostino RB Sr, Savage PJ, Levy D, et al. Trends in cardiovascular disease risk factors in individuals with and without diabetes mellitus in the Framingham Heart Study. Circulation. 2009;120(3):212-20.

Ribeiro LW, Ribeiro JP, Stein R, Leitão C, Polanczyk CA. Trimetazidine added to combined hemodynamic antianginal therapy in patients with type 2 diabetes: a randomized crossover trial. Am Heart J. 2007;154(1):78.e1-7.

Ribeiro RA, Mello RGB, Melchior R, Dill JC, Hohmann CB, Lucchese AM, et al. Custo anual do manejo da cardiopatia isquêmica crônica no Brasil: perspectiva pública e privada. Arq Bras Cardiol. 2005;85(1):3-8.

Rodríguez Padial L, Maicas Bellido C, Velázquez Martín M, Gil Polo B. Estudio prospectivo en pacientes diabéticos de la efectividad y la tolerabilidad de la trimetazidina en asociación al tratamiento previo de su enfermedad coronaria. Estudio DIETRIC. Rev Clin Esp. 2005;205(2):57-62.

Rosano GMC, Vitale C, Volterrani M, Fini M. Metabolic therapy for the diabetic patients with ischaemic heart disease. Coron Artery Dis. 2005;16(Suppl. 1):S17-S21.

Rosano G, Vitale C, Sposato B, Mercuro G, Fini M. Trimetazidine improves left ventricular function in diabetic patients with coronary artery disease: a double-blind placebo-controlled study. Cardiovasc Diabetol. 2003;2:16.

Sociedade Brasileira de Cardiologia. Diretriz de Doença Coronariana Crônica Angina Estável. Arq Brasil Cardiol. 2004;83(Supl 2):2-43.

Sociedade Brasileira de Diabetes. (2015). Diretrizes da Sociedade Brasileira de Diabetes 2014-2015. (Oliveira J, Vencio S, Eds.). São Paulo: AC Farmacêutica.

Straka RJ, Liu LZ, Girase PS, DeLorenzo A, Chapman RH. Incremental cardiovascular costs and resource use associated with diabetes: an assessment of 29,863 patients in the US managed-care setting. Cardiovasc Diabetol. 2009;8:53.

Szwed H, Sadowski Z, Pachocki R, Domzał-Bocheńska M, Szymczak K, Szydłowski Z, et al. The antiischemic effects and tolerability of trimetazidine in coronary diabetic patients. A substudy from TRIMPOL-1. Cardiovasc Drugs Ther. 1999;13(3):217-22.

Teich V, Araujo DV. Estimativa de custo da síndrome coronariana aguda no Brasil. Rev Bras Cardiol. 2011;24(2):85-94.

Thrainsdottir IS, von Bibra H, Malmberg K, Rydén L. Effects of trimetazidine on left ventricular function in patients with type 2 diabetes and heart failure. J Cardiovasc Pharmacol. 2004;44(1):101-8.

Wee Y, Burns K, Bett N. Medical management of chronic stable angina. Aust Prescr. 2015;38(4):131-7.

Werdan K, Ebelt H, Nuding S, Hopfner F, Stockl G, Muller-Werdan U. Ivabradine in Combination with Beta-Blockers in Patients with Chronic Stable Angina After Percutaneous Coronary Intervention. Adv Ther. 2015;32(2):120-37.

Xu X, Zhang W, Zhou Y, Zhao Y, Liu Y, Shi D, et al. Effect of trimetazidine on recurrent angina pectoris and left ventricular structure in elderly multivessel coronary heart disease patients with diabetes mellitus after drug-eluting stent implantation: a single-centre, prospective, randomized, double-blind. Clin Drug Investig. 2014;34(4);251-8.

Zhao P, Zhang J, Yin XG, Maharaj P, Narraindoo S, Cui LQ, et al. The effect of trimetazidine on cardiac function in diabetic patients with idiopathic dilated cardiomyopathy. Life Sci. 2013;92(11):633-8. 


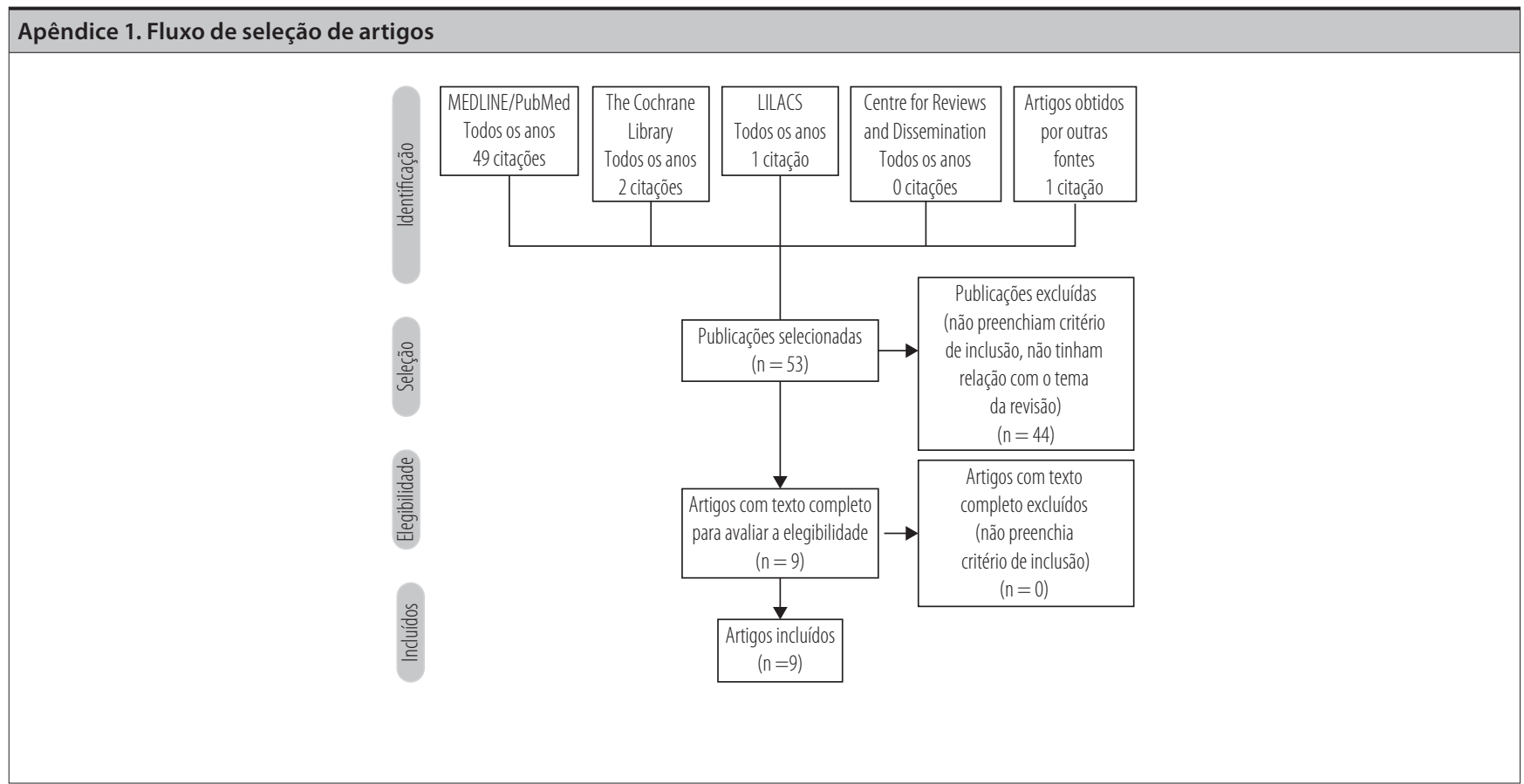

\section{Apêndice 2. Taxa de mortalidade}

\section{Taxa de mortalidade anual por outras causas}

\begin{tabular}{|c|c|c|}
\hline Idade & Taxa anual $(\%) \dagger$ & Taxa anual ajustada (\%)* \\
\hline 69 & 2,56 & 2,33 \\
\hline 70 & 2,79 & 2,54 \\
\hline 71 & 3,03 & 2,76 \\
\hline 72 & 3,30 & 3,01 \\
\hline 73 & 3,60 & 3,28 \\
\hline 74 & 3,92 & 3,58 \\
\hline 75 & 4,27 & 3,90 \\
\hline 76 & 4,65 & 4,24 \\
\hline 77 & 5,06 & 4,62 \\
\hline 78 & 5,51 & 5,02 \\
\hline 79 & 5,99 & 5,47 \\
\hline 80 & 4,68 & 4,27 \\
\hline 81 & 5,09 & 4,64 \\
\hline 82 & 5,54 & 5,05 \\
\hline 83 & 6,02 & 5,49 \\
\hline 84 & 6,54 & 5,97 \\
\hline 85 & 7,11 & 6,49 \\
\hline 86 & 7,73 & 7,05 \\
\hline 87 & 8,41 & 7,67 \\
\hline 88 & 9,14 & 8,34 \\
\hline 89 & 9,94 & 9,07 \\
\hline 90 & 10,81 & 9,86 \\
\hline
\end{tabular}

† Todas as causas.

* Taxa ajustada excluindo da mortalidade geral as mortes por doenças isquêmicas do coração. 


\begin{tabular}{|c|c|c|c|c|c|c|}
\hline \multicolumn{7}{|c|}{ Apêndice 3. Custos de tratamento } \\
\hline \multicolumn{7}{|c|}{ Custo anual do tratamento convencional } \\
\hline Medicamentos & Bula (mg/dia) & Apresentação (mg) & Custo/dose (R\$) & Doses/ano & $\%$ pacientes & Custo anual (R\$) \\
\hline AAS & 300 & 100 & 0,02 & 1.080 & 100 & 21,64 \\
\hline Clopidogrel & 75 & 75 & 0,36 & 360 & 100 & 129,04 \\
\hline Isossorbida & 40 & 40 & 0,13 & 360 & & 45,32 \\
\hline Estatinas & & & & & 100 & 991,25 \\
\hline Atorvastatina & 80 & 80 & 0,64 & 360 & & 230,65 \\
\hline Lovastatina & 80 & 20 & 2,16 & 1.440 & & $3.114,43$ \\
\hline Pravastatina & 40 & 20 & 0,80 & 720 & & 573,04 \\
\hline Sinvastatina & 40 & 40 & 0,13 & 360 & & 46,88 \\
\hline Betabloqueadores & & & & & 70,59 & 356,35 \\
\hline Atenolol & 100 & 100 & 0,05 & 360 & & 19,62 \\
\hline Carvedilol & 50 & 25 & 0,20 & 720 & & 142,42 \\
\hline Cloridrato de propranolol & 240 & 80 & 0,25 & 1.080 & & 266,71 \\
\hline Succinato de metoprolol & 200 & 100 & 1,66 & 720 & & $1.195,75$ \\
\hline Tartarato de metoprolol & 200 & 100 & 0,22 & 720 & & 157,26 \\
\hline Inibidores da ECA & & & & & 45,49 & 26,41 \\
\hline Captopril & 150 & 50 & 0,03 & 1.080 & & 37,04 \\
\hline Maleato de enalapril & 20 & 20 & 0,04 & 360 & & 15,78 \\
\hline $\begin{array}{l}\text { Antagonistas da } \\
\text { angiotensina II }\end{array}$ & & & & & 45,49 & $4.972,23$ \\
\hline Ambrisentana & 10 & 10 & 25,24 & 360 & & $9.086,00$ \\
\hline Bosentana & 250 & 125 & 8,07 & 720 & & $5.807,97$ \\
\hline Losartana potássica & 50 & 50 & 0,06 & 360 & & 22,71 \\
\hline Total & & & & & & $3.703,27$ \\
\hline
\end{tabular}

AAS = ácido acetilsalicílico; ECA = enzima conversora da angiotensina.

Os custos por grupo farmacológico foram estimados pela média dos custos dos medicamentos incluídos em cada grupo farmacológico. 
Custo anual de acompanhamento para pacientes pós-hospitalização

\begin{tabular}{|c|c|c|c|c|}
\hline Recursos de saúde & $\begin{array}{c}\text { Custo } \\
\text { unitário (R\$) }\end{array}$ & Quantidade & $\begin{array}{l}\text { Custo } \\
\text { total (R\$) }\end{array}$ & Fonte \\
\hline Consulta médica & 10,00 & 6 & 60,00 & $\begin{array}{l}\text { SIGTAP } 2016 \text { - 03.01.01.007-2 - Consulta } \\
\text { médica em atenção especializada }\end{array}$ \\
\hline Hemograma & 4,11 & 1 & 4,11 & SIGTAP 2016 - 02.02.02.038-0 - Hemograma completo \\
\hline Colesterol total & 1,85 & 1 & 1,85 & SIGTAP 2016 - 02.02.01.029-5 - Dosagem de colesterol total \\
\hline $\mathrm{HDL}$ & 3,51 & 1 & 3,51 & SIGTAP 2016 - 02.02.01.027-9 - Dosagem de colesterol HDL \\
\hline LDL & 3,51 & 1 & 3,51 & SIGTAP 2016 - 02.02.01.028-7 - Dosagem de colesterol LDL \\
\hline Triglicerídeos & 3,51 & 1 & 3,51 & SIGTAP 2016 - 02.02.01.067-8 - Dosagem de triglicerídeos \\
\hline TGO & 2,01 & 1 & 2,01 & $\begin{array}{l}\text { SIGTAP } 2016 \text { - 02.02.01.064-3 - Dosagem de transaminase } \\
\text { glutâmico-oxalacética }\end{array}$ \\
\hline TGP & 2,01 & 1 & 2,01 & $\begin{array}{l}\text { SIGTAP } 2016 \text { - 02.02.01.065-1 - Dosagem de transaminase } \\
\text { glutâmico-pirúvica }\end{array}$ \\
\hline Glicose & 1,85 & 1 & 1,85 & SIGTAP 2016 - 02.02.01.047-3 - Dosagem de glicose \\
\hline $\begin{array}{l}\text { Hemoglobina } \\
\text { glicosilada }\end{array}$ & 7,86 & 1 & 7,86 & SIGTAP 2016 - 02.02.01.050-3 - Dosagem de hemoglobina glicosilada \\
\hline Ureia & 1,85 & 1 & 1,85 & SIGTAP 2016 - 02.02.01.069-4 - Dosagem de ureia \\
\hline Creatinina & 1,85 & 1 & 1,85 & SIGTAP 2016 - 02.02.01.031-7 - Dosagem de creatinina \\
\hline Sódio & 1,85 & 1 & 1,85 & SIGTAP 2016 - 02.02.01.063-5 - Dosagem de sódio \\
\hline Potássio & 1,85 & 1 & 1,85 & SIGTAP 2016 - 02.02.01.060-0 - Dosagem de potássio \\
\hline $\begin{array}{l}\text { Radiografia } \\
\text { de tórax }\end{array}$ & 9,50 & 1 & 9,50 & SIGTAP 2016 - 02.04.03.015-3 - Radiografia de torax (PA e perfil) \\
\hline Eletrocardiograma & 5,15 & 6 & 30,90 & SIGTAP 2016 - 02.11.02.003-6 - Eletrocardiograma \\
\hline Ecocardiograma & 39,94 & 1 & 39,94 & SIGTAP 2016 - 02.05.01.003-2 - Ecocardiografia transtorácica \\
\hline Holter 24 horas & 30,00 & 1 & 30,00 & $\begin{array}{l}\text { SIGTAP } 2016 \text { - 02.11.02.004-4 - Monitoramento pelo sistema } \\
\text { Holter } 24 \mathrm{~h}\end{array}$ \\
\hline $\begin{array}{l}\text { Cintilografia } \\
\text { miocárdica }\end{array}$ & 408,52 & 1 & 408,52 & $\begin{array}{l}\text { SIGTAP } 2016 \text { - 02.08.01.002-5 - Cintilografia de miocárdio para } \\
\text { avaliação da perfusão em situação de estresse (mínimo } 3 \text { projeções) }\end{array}$ \\
\hline $\begin{array}{l}\text { Cateterismo } \\
\text { ambulatorial }\end{array}$ & 614,72 & 0,2 & 122,94 & $\begin{array}{l}\text { Quantidade: Ribeiro R. et al. 2005; Custo unitário: SIGTAP } \\
2016 \text { - 02.11.02.001-0 - Cateterismo cardíaco }\end{array}$ \\
\hline Total & & & 739,42 & \\
\hline
\end{tabular}

Custo anual de acompanhamento para pacientes sem evento prévio

\begin{tabular}{lcccl}
\hline Recursos de saúde & $\begin{array}{c}\text { Custo } \\
\text { unitário (R\$) }\end{array}$ & Quantidade & $\begin{array}{c}\text { Custo } \\
\text { total (R\$) }\end{array}$ & Fonte \\
\hline Consulta médica & 10,00 & 4 & 40,00 & $\begin{array}{l}\text { SIGTAP 2016 - 03.01.01.007-2 - Consulta } \\
\text { médica em atenção especializada }\end{array}$ \\
\hline Eletrocardiograma & 5,15 & 4 & 20,60 & SIGTAP 2016 - 02.11.02.003-6 - Eletrocardiograma \\
\hline Ecocardiograma & 39,94 & 1 & 39,94 & SIGTAP 2016 - 02.05.01.003-2 - Ecocardiografia transtorácica \\
\hline $\begin{array}{l}\text { Cintilografia } \\
\text { miocárdica }\end{array}$ & 408,52 & 1 & 408,52 & $\begin{array}{l}\text { SIGTAP 2016 - 02.08.01.002-5 - Cintilografia de } \\
\text { miocárdio para avaliação da perfusão em situação } \\
\text { de estresse (mínimo três projeções) }\end{array}$ \\
\hline Total & & 509,06 & \\
\hline
\end{tabular}

